\title{
10 AÑOS DE HISTORIA FUNDACIONAL DE LA URACCAN SIUNA-LAS MINAS
}

Fredy Leonel Valiente Contreras ${ }^{[1]}$ Víctor Manuel del Cid Lucero ${ }^{[2]}$

\section{Resumen}

La Universidad de las Regiones Autónomas de la Costa Caribe Nicaragüense (URACCAN), tiene presencia en 4 Recintos Universitarios del Caribe. En la Región Autónoma Atlántico Norte en Siuna y Bilwi; en la Región Autónoma Atlántico Sur en Bluefields y Nueva Guinea, se asimilan en relación a la estructura orgánica y líneas de trabajo: extensión, docencia e investigación.

El Recinto Siuna-Las Minas, surge en 1995 para dar respuesta a una demanda histórica de siglos de exclusión económica, política, cultural y social por parte del Estado nacional nicaragüense. El objeto de esta sistematización fue rescatar la historia y la generación de propuestas alternativas en la construcción de la autonomía de la Costa Atlántica. Es de tipo longitudinal de 1995-2005 y se realizó en el 2007 a partir de un enfoque cualitativo básicamente con entrevistas In situ individuales y grupales, además se estudiaron las memorias de los talleres, actas, resúmenes de los debates, datos estadísticos, evaluaciones y diagnósticos institucionales.

Con un enfoque sistémico se ha concluido que en el ámbito nacional la URACCAN por su perfil multiétnico, intercultural bilingüe y su praxis de acompañamiento comunitario contribuye al desarrollo identitario en la forja de la Autonomía de la Costa Caribe donde conviven seis culturas: miskitu, sumu-mayangna, rama, garífuna, creol y los pueblos del mestizaje.

Palabras clave: Sistematización, URACCAN Recinto Siuna-Las Minas, autonomía.

\section{Summary}

The University of the Autonomous Regions of the Caribbean Coast of Nicaragua (URACCAN), is present in four Campuses. In the North Atlantic Autonomous Region in Siuna and Bilwi; and in the South Atlantic Autonomous Region in Bluefields and Nueva Guinea, as they are treated in relation to the organizational structure and working lines: extension, teaching and research.

\footnotetext{
${ }^{[1]}$ Profesor universitario en Arte, Letras, Sociología y Filosofía. Editor de la revista Ciencia e Interculturalidad de la URACCAN. fredvaliente@yahoo.com

[2] Máster en Antropología y educador popular. delcidlucero@yahoo.com
} 
The Siuna Campus was founded in 1995, in response to a historical demand of centuries of economic, political, cultural and social exclusion by the Nicaraguan national state. The purpose of this systematization was to rescue the history and the generation of alternative proposals for the construction of the autonomy of the Atlantic Coast. It's a longitudinal study from 1995-2005 and it was carried-out in 2007 from a primarily qualitative approach with individual and group interviews in situ; we also examined the reports of the workshops, the minutes, summaries of discussions, statistics, evaluations and institutional diagnostics.

It was concluded -using a systemic approach- that URACCAN at a national context and due to its multiethnic and intercultural-bilingual profile, as well as it community accompaniment praxis contributes to identity development in forging the autonomy of the Caribbean Coast where six cultures coexist: Miskitu, Sumu-Mayangna, Rama, Creole, Garifuna, and Mestizo.

Keywords: Systematization, URACCAN-Siuna Campus, autonomy.

\section{Introducción}

Sólo la ficción sabe, sólo la imaginación puede tocar, con la punta de los dedos, un retazo de verdad. DANTE LIANO (El misterio de San Andrés)

\section{Las Regiones Autónomas de la Costa Caribe Nicaragüense}

Nicaragua tiene 130,334. 54 Km2 de extensión territorial. La Región Autónoma Atlántico Norte (RAAN) y la Región Autónoma Atlántico Sur (RAAS), tienen una extensión de 6o, $366 \mathrm{Km} 2$ que equivalen al 50 \% de la superficie total del país. Las Regiones Autónomas se instituyeron en 1987 conforme a la Ley No. 28, Estatuto de Autonomía de las Regiones de la Costa Atlántica; asimismo, la Ley de Lenguas, No. 162, y la Ley No. 445, reconocen los derechos de los pueblos indígenas y comunidades étnicas en la región, quienes tradicionalmente conviven en el territorio mucho antes de la colonización europea en 1492.

La población estimada es de 620,640 habitantes y su mayoría habita en áreas rurales, según el VIII Censo de Población y IV de Vivienda del año 2005, la RAAN tiene aproximadamente 314, 13 o habitantes, con una densidad poblacional de 9.5 habitantes por kilómetro cuadrado. La RAAS tiene 306, 510 habitantes aproximadamente y una densidad poblacional de 11. 2 habitantes por $\mathrm{Km} 2$.

La RAAN comprende los municipios de Waspam, Puerto Cabezas, Rosita, Bonanza, Waslala, Siuna, Prinzapolka y Mulukukú. El $42 \%$ de su población corresponde a la etnia mestiza, el $40 \%$ a la etnia miskitu, $10 \%$ a la creole y el $8 \%$ sumu-mayangna. La principal ciudad de la Región Autónoma Atlántico Norte es Bilwi, Puerto Cabezas. 
El municipio de Siuna se encuentra localizado a $318 \mathrm{Km}$, al noroeste de la Ciudad Capital, Managua. El suroeste de la RAAN es denominado Triángulo Minero y comprende los municipios de Siuna, Rosita y Bonanza. La mayoría de sus habitantes se dedican a la explotación minera, forestal y en menor grado a las actividades pecuarias y productivas. Según el Informe de Desarrollo Humano del 2005 (PNUD), el índice de pobreza alcanza el $83.2 \%$ considerado como pobreza extrema, un $12.1 \%$ pobres y el 4. $6 \%$ de no pobres.

Las comunidades del Caribe han desarrollado sus asentamientos humanos en las dinámicas del mercado y en los contextos de las invasiones y colonización metropolitana -española, inglesa y norteamericana-, pasando por la anexión del territorio de la Reserva de la Mosquitia al Estado nacional nicaragüense en 1894, hasta el momento actual. El resultado de los procesos migratorios junto a las políticas de ocupación e integración forzada, aunados a los conflictos en décadas pasadas y al desplazamiento de la frontera agrícola ha dado lugar a una profunda transformación biodemográfica en los últimos tres lustros.

Los municipios de Siuna, Bonanza y Rosita localizados en el llamado "Triángulo Minero", en la RAAN, son conocidos por su importancia como enclaves mineros durante el siglo veinte, administrados por empresas norteamericanas como la Rosario Mining Company y La Luz Mining Company, que explotaron enormes cantidades de oro, hierro y otros minerales que desde puerto "Isabel" en Alamikamba, se embarcaban hacia los Estados Unidos. En la segunda mitad del siglo pasado, Nicaragua se ubicó entre los países con mayor producción aurífera a nivel mundial.

Actualmente en Siuna en el sector de La Luz, se mantienen a punto de derrumbarse antiguas instalaciones del complejo minero. Se dice que la ciudad actual está construida sobre una red de túneles inundados de agua. De la época dorada sólo quedó un sabor a tristeza y la añoranza de los comisariatos de las empresas, la silicosis en los pulmones de los mineros mestizos y miskitus que se adentraban a diario en los profundos túneles. En Bonanza continua la explotación minera, ahora por la empresa canadiense Hemco.

La población ya no es la misma de aquellos años, los mineros miskitus regresaron a sus pueblos y en el barrio Mosquitown viven otras personas, igual en Jumeca, barrio donde vivían las antiguas familias creoles. Son otros tiempos, son ganaderos y campesinos mestizos la mayoría de la población de Siuna, donde solamente hay una población indígena sumu-mayangna en Sikilta. En Bonanza, la Reina de las Montañas, se concentra la mayoría de la población Sumu-Mayangna, así como en otras comunidades de Rosita. En los municipios de Waslala y Mulukukú, la mayoría de la población es mestiza, llegada en oleadas migratorias en las últimas décadas. 
Según el Censo Nacional del año 2005, la población de los municipios de Rosita $(22,723)$, Bonanza $(18,633)$, Waslala $(49,339)$, Mulukukú $(29,838)$ y Siuna $(64,092)$, es equivalente al $58 \%$ del total de la Región Autónoma del Atlántico Norte, RAAN. Su creciente población es de la etnia mestiza, con una minoría sumu-mayangna. La economía de esta sub-región está basada en la ganadería semi-intensiva, la minería industrial y artesanal, explotación forestal y el comercio. Grandes extensiones de bosque han sido convertidas en pastizales, amenazando la frontera agrícola-ganadera con extenderse al territorio de la reserva de la biósfera de Bosawas. En este contexto histórico y geocultural de crisis y desafíos sociales se empezó a gestar el sueño de tener un centro de estudios superiores donde pudieran, por fin, estudiar los adultos y la juventud de los municipios del Triángulo Minero.

Esta necesidad marca el hito histórico de fundar la URACCAN Recinto Siuna-Las Minas, afín de facilitar procesos técnicos y científicos que coadyuven a la población, en un ambiente de diálogo intercultural, a la construcción de la autonomía, por lo que es imprescindible determinar los procesos históricos comprendidos en la década de 1995 al 2005, etapas que han permitido la fundación, desarrollo y consolidación de la URACCAN. Este autoconocimiento permitirá constituirse en guía para la reflexión y acción participativa de la comunidad académica y de la población costeña para seguir generando procesos de empoderamiento que propicien la plena igualdad política, económica y cultural en el marco de los derechos humanos, la Constitución de la República y la Ley de Autonomía. Esta sistematización se hizo ante el vacío histórico en el marco referencial de su identidad constitutiva para saber por qué surgió, cuándo, dónde, cómo y cuál es su importancia.

\section{LA URACCAN un puente en la construcción de Autonomía en las Regiones Autónomas}

La Autonomía es el derecho de las personas y comunidades a decidir por sí mismas y a construir para sí el tipo de vida deseado. La autonomía tiene dos dimensiones principales: por una parte el derecho a una cultura e identidad propias, lo cual incluye: memorias, tradiciones, lenguas y el derecho a la tierra; por la otra, el derecho a la autodeterminación política de la comunidad organizada democráticamente.

El artículo No. 15 de la Ley de Autonomía de las Regiones Autónomas, Ley No. 28, establece que dentro de sus territorios, en consonancia con la Constitución Política de la República de Nicaragua, el Gobierno Regional está conformado por el Consejo Regional y los siguientes órganos de administración: Consejo Regional y Gobierno Regional, autoridades municipales y comunales y otros correspondientes a la subdivisión administrativa de los municipios. En este marco jurídico se adscribe la URACCAN con el objeto de contribuir a la construcción de la Autonomía de la Costa Atlántica. 


\section{Revisión de literatura}

URACCAN (2007). Sistematización del proceso de creación y desarrollo de la URACCAN en el triángulo minero 1995-2005. Esta tuvo lugar In situ con los protagonistas del hecho fundacional y la consulta del archivo documentario. Asimismo, en cuanto a la bibliografía pertinente a la metodología de la sistematización se han valorado específicamente sobre el rescate de las experiencias y las alternativas sistémicas para la innovación sinérgica.

\section{Materiales y método}

Grabadora, registros documentales y bitácora de las descripciones desde una perspectiva de relectura simbólica. Es de corte longitudinal, abarca el período 1995-2005. La sistematización fue realizada en el 2007 a partir de un enfoque cualitativo básicamente con entrevistas a los protagonistas, estudio de las memorias de los talleres, actas de reuniones, resúmenes de los debates, informe de investigaciones, manuales de capacitación, evaluaciones y diagnósticos institucionales.

\section{Resultados y discusión}

\section{El Contexto educativo en las Regiones Autónomas de la Costa Caribe}

La educación en las Regiones Autónomas de la Costa Caribe de Nicaragua se caracteriza por el proceso de construcción y fortalecimiento de la Autonomía. Este proceso autónomo en la Educación Superior se manifiestan en la existencia de dos centros universitarios: la Universidad de las Regiones Autónomas de la Costa Caribe Nicaragüense (URACCAN) y la Bluefields Indian and Caribean University (BICU).

La Educación Primaria y Secundaria está en proceso de reformulación y adecuación curricular a la realidad multi e intercultural de la población costeña a través del Subsistema Educativo Autonómico Regional (SEAR), constitutivo del Plan Nacional de Educación y del Programa de Educación Bilingüe Intercultural (PEBI).

Sin embargo, por las características geográficas, climáticas, sociales, económicas y culturales, asimismo la caótica infraestructura de transporte y comunicación, el acceso a la Educación Secundaria en los municipios y comunidades rurales es sumamente precario y casi inexistente; a la vez la demanda de docentes y centros educativos se ha incrementado por el aumento de la población, sobre todo a lo largo de la frontera agrícola y ganadera.

La descentralización del sistema educativo, la asignación de recursos educativos a las instancias regionales y un apoyo efectivo al Subistema Educativo Autonómico Regional (SEAR), son pasos decisivos para mejorar ese difícil panorama. 
En búsqueda de soluciones en el ámbito educativo, la Educación Primaria y Secundaria en las comunidades rurales se realiza bajo el sistema multigrado. En muchas de las comunidades, ante la falta de plazas para maestros, las iglesias, Morava especialmente, y los padres de familia asumen por su iniciativa los costos de la educación de sus hijas e hijos.

\section{Proceso fundacional de la URACCAN}

En 1976-1977, se hizo el primer intento para desarrollar la Educación Superior en la Costa Caribe con la apertura de dos núcleos de la UNAN-Managua, uno en Puerto Cabezas y el otro en Bluefields. Los estudiantes de Las Minas asistían al núcleo de Puerto Cabezas, donde junto a los condiscípulos de Waspam y Puerto Cabezas recibían clases en el Convento de las Monjas Carmelitas. Esta primera experiencia fue interrumpida por la lucha revolucionaria y el posterior triunfo de la Revolución Popular Sandinista el 19 julio de 1979.

En el período del gobierno revolucionario se reanudan las gestiones con los dirigentes de la Educación Superior: Sergio Ramírez, vicepresidente de la república quien presidía el Consejo de Educación; el Dr. Joaquín Solís Piura, presidente del Consejo Nacional de Educación Superior (CNES); Dr. Alejando Serrano Caldera, rector de la UNAN-Managua y el Padre Fernando Cardenal, Ministro de Educación. Fruto de estas gestiones, se realiza el Programa Universitario de Educación a Distancia (PRUEDIS), con una metodología diseñada por la UNAN-Managua y con el apoyo de docentes de la República de Cuba.

Con el decreto publicado el 30 de octubre de 1987 en La Gaceta Diario Oficial, entra en vigencia el Estatuto de Autonomía de las Regiones de la Costa Atlántica de Nicaragua, garantizando así el ejercicio de los derechos de los pueblos indígenas, afrodescendientes y comunidades mestizas a la salud y Educación Intercultural, derechos a su lingualidad, laborales, a la tierra, sus tradiciones y costumbres. En virtud de este decreto se logra la creación de la Universidad de las Regiones Autónomas de la Costa Caribe de Nicaragua (URACCAN), autorizada a funcionar por la resolución del Consejo Nacional de Universidades (CNU) con fecha del 5 de marzo de 1992, y con personería jurídica otorgada por la Asamblea Nacional el 3 de junio de 1993. Se publica en la Gaceta, Diario Oficial No. 104, tres de junio de 1993, decreto No. 602.

Se autodefine como Universidad Comunitaria Intercultural de los pueblos indígenas y afrodescendientes. El concepto de Universidad Comunitaria se inscribe en el marco del derecho internacional en reconocimiento de los derechos humanos. De igual manera, promueve la cosmovisión y cosmogonía de los pueblos indígenas constitutivos de una energía revitalizadora de saberes y conocimientos, que son claves en los procesos propios de auto desarrollo, es decir, el legítimo derecho que poseen los pueblos de configurar su futuro en un marco de autonomía, de justicia y oportunidad de igualdades políticas, económicas, sociales y culturales. 
Misión: Contribuir al fortalecimiento del proceso de Autonomía de la Costa Caribe de Nicaragua, por medio de la capacitación y profesionalización de recursos humanos, dotándolos de los conocimientos necesarios para conservar y aprovechar de forma racional y sostenida los recursos naturales.

Visión: La URACCAN es la universidad intercultural de los pueblos indígenas y comunidades étnicas de la Costa Caribe nicaragüense, que fortalece la Autonomía a través del acompañamiento de procesos de autogestión, unidad multiétnica y la formación integral de mujeres y hombres costeños.

Los ejes transversales de la URACCAN son: el género, a partir de las relaciones dialógicas y de respeto entre mujeres y hombres; la interculturalidad como valoración a las diversas culturas, el desarrollo autosostenible que se promueve en el acompañamiento humano y técnico a las comunidades y este es el principio rector en la Política de Extensión Social y Comunitaria.

La Investigación: En la URACCAN se promueve un proceso participativo de generación de conocimientos y capacidades, revitalización, respeto a los saberes locales, y a las experiencias de los pueblos indígenas y comunidades afrodescendientes, en búsqueda de transformar y desarrollar el proceso autonómico regional.

Con la Política de Extensión Social y Comunitaria se facilita la vinculación de las comunidades con su liderazgo, las autoridades comunitarias, municipales y regionales, las organizaciones sociales, con el objeto de lograr un mejor beneficio de los recursos y capacidades locales.

\section{La Asociación Pro-URACCAN}

A fin de materializar la idea de la universidad caribeña, surge la Asociación ProURACCAN, constituida según el acta del 13 de abril de 1991 y liderada por once personalidades: el Dr. Ray Hooker Taylor, decano de la Facultad de Humanidades de la Universidad Nacional Autónoma de Nicaragua UNAN-Managua, la Dra. Mirna Cunningham Kain y el Ing. Albert StClair (en representación de la RAAN); Lic. Hugo Sujo, Dr. Roberto Hodgson Joseph, Dr. Hennigston Omeir Webster, Dr. Cyril Omeir Green, Licda. Mirna Viales Álvarez, Dr. Alfredo Arana Cantero, Revda. Doris Merlin Forbes Harvey, Dr. Alfredo Cordero Cabrera y Licda. Debora Hodgson (en representación de la RAAS), quienes gestionaron la personería jurídica ante el Consejo Nacional de Universidades y la Asamblea Nacional. A este grupo se incorporan posteriormente Francisco Cambell, Sandra Hooker y Daisy George.

Apoyaron desde el principio el poeta David McFields y el profesor Guillermo McLean y entre los estudiantes: Yuri Zapata Web (Puerto Cabezas), Ángela Fletes 
(Bluefields) y Jacoba Dávila (Las Minas), quienes representaban a la Asociación de Estudiantes de la Costa Atlántica (AEESCA). En representación de los municipios del Triángulo Minero: José Antonio Mairena y Fidencio Devis de Bonanza; Oscar Ríos de Siuna y Fernando Espinoza de Rosita (q. e. p. d). Para apoyar la gestión de la Asociación Pro-URACCAN, en septiembre de 1994 se conformó un Comité en Las Minas, con la participación de Pío Alejandro García Izaguerri, Benito Talavera, Rodrigo Larrave, Beanira Agüero, Mirna Campbell, Ana Lazo, Alejandra Centeno, Oscar Ríos, Fernando Jerez, Manuel Marín y Remigio Hernández Alemán, alcalde del municipio de Siuna en esa época.

\section{La segunda edición del PRUEDIS}

En 1995 se logró la cooperación del Fondo de Asistencia Internacional de los Estudiantes y Académicos de Noruega (SAIH) para implementar una segunda edición del PRUEDIS durante el período 1995-1997 con la coordinación de FADCANIC y el apoyo de la UNAN-Managua, la URACCAN graduó a 102 profesionales de Ciencias de la Educación en ambas Regiones Autónomas.

El PRUEDIS II atendía simultáneamente a estudiantes de las 2 Regiones Autónomas en las áreas de Ciencias de la Educación: Geografía, Historia, Física, Matemáticas, Español e Inglés. Los estudiantes obtuvieron los títulos de Profesores en Educación Media, meta del programa. En esta segunda etapa se incorporan docentes originarios de la Costa, entre los cuales podemos mencionar a Marlene Chow, Aleyda Chow y Stern Robinson.

\section{Contexto de fortalecimiento institucional de la URACCAN Recinto Las Minas}

El contexto internacional está marcado por el neoliberalismo que conduce a la extrema miseria a las clases subordinadas y esta es la corriente que priva en Nicaragua y la subsecuente cadena de clientelismo y corrupción; en la RAAN, en los municipios del Triángulo Minero el decenio 1995-2005, se caracteriza por las recurrentes crisis energéticas, el detrimento y falta de mantenimiento de las carreteras, la falta de inversión y estrategias para el desarrollo justo e integral, la inseguridad ciudadana y proliferación de grupos fuertemente armados, el avance incontrolable de la frontera agrícola, el acoso de los colonos mestizos sobre la reserva de la biósfera en Bosawas, los intentos de crear un nuevo departamento en Las Minas y la creciente deforestación de los bosques.

Aun contra corriente, los pueblos indígenas y afrodescendientes mantienen su lucha emancipadora y se generan significativos avances en el proceso de Autonomía, como es la Reglamentación de la Ley No. 28 o Estatuto de Autonomía, el desarrollo del Sistema Educativo Autonómico Regional (SEAR), promulgación de la Ley No. 445 o de Demarcación Territorial. En los municipios de Siuna, Rosita, Bonanza y Waslala, 
con la cooperación austriaca a través del organismo Horizontzooo se consolida la URACCAN, al proveer de recursos humanos calificados y un programa de investigaciones para generar y proponer estrategias para el desarrollo humano autosostenible y con principios de equidad ciudadana.

En síntesis, el desarrollo organizacional del Recinto Siuna-Las Minas, parte de una etapa preparatoria para su lanzamiento desde 1994 en que surge la idea de fundarla y las etapas de reestructuración y afianzamiento de las experiencias hasta el 2005.

\section{Factores dinamizadores}

Los docentes y el vicerrector Ing. Bismarck Lee han manifestado que la organización de las actividades universitarias está ligada a los factores como el uso apropiado de los marcos jurídicos establecidos, el liderazgo articulado de las instituciones, la noción del bien común institucionalizado en el paradigma comunitario y el papel de las mujeres como articuladoras y garantes del capital social, la revitalización de la lengua y la cultura, la problemática de la educación formal e informal, los intereses y expectativas económicas, el referente de modelos autóctonos, el rol de los partidos políticos y el despliegue de los intereses de las comunidades.

\section{Factores distorsionadores}

Dentro la dinámica de organización se han valorado los factores distorsionadores entre los que se mencionan las secuelas de reasentamiento de la población en tiempos de la guerra y la educación sin pertinencia cultural, la ausencia de escuelas de secundaria en el área rural y el abandono de las escuelas primarias, la violencia visible y encubierta en el "código patriarcal" que rige la vida cotidiana en el mundo rural, el consumo del alcohol y el trasiego de drogas.

\section{Dinámica de los procesos internos de la organización}

La fundación del Recinto URACCAN Siuna-Las Minas, implicó: organización de la oferta académica, selección del personal, infraestructura, creación y atención a las extensiones universitarias, seguimiento académico y financiero; currículum de las asignaturas, modalidad de las carreras, planificación y cronograma docente, , bibliografía, convocatoria, financiamiento interno y externo, aprobación de las carreras a nivel oficial, nóminas y cancelación de honorarios de conformidad a partida presupuestaria, definición de estatutos internos, manual de funciones de los funcionarios, contratación de personal de servicio administrativo, y todas aquellas actividades previas de organización e investigación para definir las carreras. 


\section{LA URACCAN en el Triángulo Minero}

En septiembre de 1994 se estableció el Recinto Las Minas en el municipio de Siuna, la decisión de su ubicación se debió a que, geográficamente es la puerta de entrada a la RAAN; además de presentar criterios tales como: mayor población estudiantil apta para la Educación Superior, mayor actividad económica, presencia de organismos gubernamentales, no gubernamentales y centros educativos de secundaria. Participaron en estas reuniones preparatorias, por el municipio de Bonanza José Antonio Mairena Malespín y Eugenio Pao; por Rosita, Fernando Espinoza (q. e. p. d. ) y por Siuna, Oscar Ríos Palacios y Beanira Agüero.

En noviembre de 1994, ante el Párroco Wilberto Lancer, se obtuvo el préstamo de aulas de clase en el Colegio Parroquial "Maura Clarke". En 1995, se realiza la matrícula oficial de 152 estudiantes en las carreras de Ingeniería Agroforestal, licenciaturas en Administración de Empresas con mención en Recursos Naturales y en Sociología con mención en Autonomía. Los primeros estudiantes fueron hombres y mujeres de mediana edad, directivos de las instituciones del Estado y entidades privadas de desarrollo social.

El cuerpo docente se conformó con profesionales autóctonos del municipio, entre ellos: Brunilda Campos, Beanira Agüero, Mario Ríos, Carlos Madrigal y Haydeé Wilson, quienes cumplían con los requisitos exigidos: ser autóctono de la región, tener un título profesional, identificados con la Autonomía de las Regiones Autónomas.

\section{Fundación y desarrollo: 1995-1996}

En el marco de las dificultades y desafíos de los contextos internacionales, nacionales, regionales y al interior de la universidad, el resultado es que en 1995 se logra inaugurar la URACCAN, con sede en Siuna. Se apertura con el primer año de estudios generales con: 6 docentes, 1 vicerrector, 1 secretaria, 1 administrador. Se iniciaron las clases en la modalidad regular con tres grupos: 1 matutino y 2 nocturnos. Las carreras ofertadas: Administración de Empresas, Sociología con mención en Autonomía e Ingeniería Agroforestal. Se obtuvo una población estudiantil de 152 estudiantes originarios de los municipios de Rosita, Bonanza y Siuna.

Desde su fundación, el Recinto Siuna-Las Minas obtuvo el apoyo del organismo austriaco Horizont30oo (antes OED), en lo relacionado al pago del personal docente. Hasta el año 1997, con la inclusión de URACCAN en el Consejo Nacional de Universidades (CNU), el pago de docencia fue asumido con el presupuesto del $6 \%$ y también incluyó becas a estudiantes de escasos recursos económicos.

Los miembros de la Asociación Pro-URACCAN en Siuna, fueron el Ing. Manuel Marín Castellón, vicerrector; Arsenio Montalbán, administrador; Blanca Centeno, 
secretaria; docentes: Mario Ríos, Matemática; Beanira Agüero, Recursos Naturales; Brunilda Campos, Español; Carlos Madrigal, Historia; Sonia Salmerón, Conserje; Florencio Reyes, Vigilante. Se obtuvo un local prestado por la parroquia católica.

En cuanto a la tecnología, se reducía a una eficiente máquina de escribir y un mimeógrafo, el único existente en el Triángulo Las Minas. Entre esténciles, resmas de papel y tubos de tinta, la señora Sonia Salmerón reproducía los documentos de apoyo didáctico. En 1996, la novedad en Siuna fue la adquisición de una computadora con el programa Word Perfect 5. 1 y una impresora matricial de las que usan cinta. Desde ahí Leonor Ruiz, mantenía el Registro Académico, mientras la observaban desde lejos, como cuando en Cien Años de Soledad el prestidigitador lleva la primera lupa a Macondo. A toda esta tecnología de punta, se sumaba un proyector de acetatos. En 1996, el Recinto Las Minas incrementó sus actividades académicas por lo que se contrató a seis nuevos docentes: Mercedes Godoy, Juliana Francis, Wilfredo Romero, Guillermina Solórzano (mexicana), Martín Erants, Verónica Valdivia y Arsenio Montalván, conformando un cuerpo docente de once catedráticos.

También se adquiere un área de 11. 7 hectáreas para el recinto, donación por parte del Sr. Freddy Figueroa, originario de Boaco. Luego, se obtuvieron fondos de SAIHNoruega para la construcción del primer edificio, que es donde actualmente funciona la biblioteca. Asimismo, se trabaja en la creación del Instituto de Recursos Naturales, Medio Ambiente y Desarrollo Sostenible (IREMADES).

\section{Apertura de extensiones universitarias en Rosita, Bonanza y Waslala}

En noviembre de 1995, abren sus puertas las extensiones universitarias en Rosita y Bonanza y se oferta la cátedra de Género. En 1996, la URACCAN es incluida en la Ley de Autonomía de la Educación Superior y se fortalece la institucionalidad con la aprobación de los Estatutos de la Asociación y la definición de líneas de acción.

En 1996, se inicia el desarrollo académico en las extensiones universitarias de Rosita y Bonanza. Para el desarrollo de las clases se contratan docentes del Pacífico por no disponer del suficiente personal en la zona. Se contratan docentes que viajaban desde Siuna a facilitar clases a las extensiones universitarias. Se disponía de un personal docente de 12 integrantes contratados a tiempo completo y se apertura en Rosita y Bonanza el año de Estudios Generales. Quienes se encargan del proceso son: un coordinador y 4 docentes autóctonos; se inaugura la Modalidad Sabatina con el propósito de ofrecer la carrera de Ciencias de la Educación con mención en Ciencias Naturales y Ciencias Sociales afín de formar profesores de Educación Media (PEM), y el ciclo diversificado para maestros normalistas con necesidad de profesionalización, los principales protagonistas son miembros de la Asociación Pro-URACCAN: Fernando Espinoza, José Antonio Mairena, Fidencio Devis, Oscar Ríos y Jacoba Dávila. 
En el período 1997-2000, el vicerrector Manuel Marín Castellón realiza sus estudios de maestría y entra a fungir como vicerrectora interina la licenciada Thelma Sánchez hasta 1999. Se realiza el registro y publicación de estatutos, adquisición de instalaciones propias en Siuna y Rosita. Se descentralizan los fondos económicos a los institutos de investigación, se genera mayor cooperación externa. En 1999, se realizaron acciones encaminadas al fortalecimiento de las capacidades pedagógicas de los docentes en el sector minero: Rosita, Bonanza, Siuna, a través de la apropiación de las metodologías activas y participativas, proceso facilitado por la ingeniera colombiana: Bárbara Kraussen, asesora técnica de Bosawas-Siuna, así como la Planificación Universitaria facilitada por la MSc. Amanda Puhiera, Secretaria Académica de la URACCAN.

Se ofertaron dos modalidades, en cursos regulares: Ingeniería Agroforestal, Administración de Empresas y licenciatura en Sociología con mención en Autonomía; en la modalidad de sabatinos el Ciclo Básico y el Bachillerato en Ciencias y Letras. En la extensión de Rosita, la modalidad sabatinos se ofertó el año de estudios generales y Ciencias de la Educación y por encuentro la carrera de Contabilidad; en la extensión de Bonanza en sabatinos, Ciencias de la Educación.

\section{Hacia el fortalecimiento institucional}

En 1998 se amplía la planta docente y se realiza transformación curricular en Ingeniería Agroforestal. Ingresan 6 nuevos docentes: Julia Argüello, Aidalina Balladares, Iris López, Octavio Rocha, Marvin Chávez y Víctor Ronald Zúniga. En el 2,ooo se concreta el proyecto trianual de fortalecimiento institucional, se inicia el proceso para formular el segundo proyecto para el período 2001-2003. Entre los componentes se inicia el proyecto de la Preparatoria con 102 estudiantes de las comunidades rurales de Siuna, Rosita y Bonanza. Se formula el primer proyecto de fortalecimiento institucional 1998-2000, e incluye cuatro componentes básicos: a) capacitación, b) programa de becas, c) infraestructura y d) bibliografía.

El personal docente tanto horario como permanente atendió para el año 2000 la cantidad de 423 estudiantes de los cuales 268 eran mujeres y 155 varones. Debido al incremento de la matrícula y al desarrollo de la universidad, se hace necesaria la creación de las coordinaciones de carrera: el ingeniero Bismarck Lee por Agroforestal, la licenciada Jacoba Dávila en Sociología y la licenciada Aidalina Balladares en Administración de Empresas.

En el 2001 es electo vicerrector el ingeniero Bismarck Lee, ingresan 444 estudiantes de los cuales 238 eran mujeres y 206 varones. Existe una mayor representación de las etnias, ya que ingresan estudiantes miskitus y mayangnas en las diferentes carreras. También se cambia al coordinador de Ingeniería Agroforestal, asumiendo el Ing. Victor Zúñiga y la licenciada Aura González, asume como nueva coordinadora de Administración de Empresas. Se apertura la carrera de Ingeniería en Zootecnia en 
donde participan los médicos veterinarios: Letisia Castillo e Iván Jarquín y la Inga . Carmenza Chang.

Se establece el Instituto de Estudios y Promoción de la Autonomía (IEPA- Las Minas) coordinado por el licenciado Octavio Rocha (q. e. p. d. ). En julio se crea la Radio Comunitaria URACCAN- La Voz del Saslaya 94. 1 FM, financiada por Oxfam-Gran Bretaña y coordinada por Julia Argüello. Se organiza el primer Simposio Internacional sobre Biodiversidad, Propiedad Intelectual y Derechos Indígenas organizado por IREMADES. En infraestructura, se construyen los módulos para las oficinas administrativas, sala de docentes, la residencia estudiantil para albergar a los becados y el comedor. Con el acompañamiento de Horizontzooo se estableció una extensión universitaria en Waslala en el año 2002.

En el 2002, asume el Ing. Jamill Castillo la coordinación de la carrera de Ingeniería Agroforestal, la Dra. Letisia Castillo en Zootecnia y la Licda. Aura González en Administración de Empresas. En cada una de las carreras se exige syllabus, avances programáticos, proyectos de exámenes que se registran en expedientes. Ingresan 427 estudiantes (251 mujeres y 176 hombres). 14 miskitu, 44 mayangnas, 3 creole, 204 mestizos de los cuales 101 son mujeres y 103 hombres. Asimismo, egresan 151 estudiantes: 73 mujeres, en diferentes niveles académicos.

En el 2003, se convoca a las elecciones de Vicerrectoría y participan dos profesionales: el Ing. Bismarck Lee y el Lic. Pío Alejandro García Izaguirre. Resulta electo por decisión unánime en el Consejo Universitario de Recinto, el Ing. Bismarck Lee.

Permanece el proceso de autoevaluación institucional de cara a la acreditación universitaria. Asimismo, se impulsa la transformación curricular en las carreras de Ingeniería Agroforestal y Administración de Empresas, con la finalidad de actualizar y estandarizar el plan de estudios en toda la universidad y participaron todos los docentes; se instala el servicio de Internet con la finalidad de garantizar información actualizada y contribuir con la calidad de la formación profesional.

\section{Una universidad comunitaria e intercultural fortalecida}

El período 2000-2005 se caracteriza por el fortalecimiento institucional. Se realizan elecciones para rectoría y es elegida la MSc Alta Hooker Blandford (2003 - 2007). Se institucionaliza el Consejo Universitario de Recinto (CUR) donde están representados los gremios docentes, estudiantiles y trabajadores; se consolida el modelo de Universidad Comunitaria Intercultural. Se realiza la adecuación curricular de las carreras de Administración de Empresas, Ingeniería Agroforestal y Sociología con mención en Autonomía e Ingeniería en Zootecnia. En el 2002 se promueve el Centro 
de Estudios e Investigación de la Mujer Multiétnica (CEIMM) con la coordinación de la MSp. Jacoba Dávila.

\section{La oferta académica en las extensiones universitarias Siuna-Las Minas}

\section{Oferta académica 2000}

En la extensión universitaria de Rosita se ofertan 5 carreras. Se apertura el primer año de Estudios Generales para la carrera de Español y primer año de Contabilidad. Se mantuvieron dos grupos en Ciencias Sociales con mención en Historia en IV y V año respectivamente. Un $V$ año de Biología. Se obtuvo una matrícula de 113 estudiantes, según las estadísticas y se obtuvo un $80 \%$ de retención escolar y un rendimiento académico del 92\%, lo cual refleja los niveles cualitativos alcanzados en la universidad producto de su visión y su misión, de los nuevos enfoques pedagógicos y metodológicos.

\section{Oferta académica 2001}

En el primer semestre del 2001 se inició en la extensión universitaria de Rosita el primer año de Técnico Superior en Desarrollo Municipal dirigido a personal administrativo y político de las Alcaldías de Rosita, Siuna, Bonanza y Prinzapolka, en el marco de fortalecer el desarrollo de las Regiones Autónomas. Se ofertaron las carreras de CCSS con mención en Desarrollo Local, Español II año, V año Historia, Educación Intercultural Bilingüe (EIB, primera generación) y un diplomado en Comunicación Social.

\section{Oferta académica 2002}

En la Extensión de Rosita se ofertaron las carreras de Ciencias de la Educación con mención en Español para obtener el título de Profesores de Educación Media. Asimismo, se concluyó el tercer semestre en la carrera de Ciencias Sociales con mención en Desarrollo Local.

\section{Oferta académica 2003}

Se dio inicio a la carrera de Pedagogía con mención en Educación Primaria, la carrera de Educación Intercultural Bilingüe en dos niveles, Técnico Superior y Licenciatura. En 2003 se ofertaron cinco carreras: Ciencias Sociales con mención en Desarrollo Local, Español, Pedagogía con mención en Educación Primaria y Educación Intercultural Bilingüe (EIB).

Asimismo, se realizó la tercera graduación de Técnico Superior. Se graduaron 17 profesores en Ciencias de la Educación con mención en Español. De este grupo, un estudiante pertenecía a la etnia mayangna, el resto fueron mestizos. Se realizó la primera graduación de Técnico Superior en Desarrollo Municipal: 9 mujeres y 14 
hombres, para un total de 23 estudiantes. De este grupo, 2 eran mayangnas, 1 de la etnia miskitu y el resto mestizos.

Se realizó la primera graduación en las licenciaturas en Ciencias de la Educación con menciones en Historia y Biología. En general, esta fue la tercera graduación de Técnicos Superiores y la primera en Ciencias de la Educación.

También se adquirió un terreno de 1. 5 manzana para la construcción de la nueva infraestructura donde se ubicaría la extensión universitaria en el municipio de Rosita.

\section{Oferta académica 2004}

En el 2004 se gradúan los primeros Magister en el recinto con una maestría en Docencia Universitaria, se ofertaron 7 programas docentes de postgrado y 8 licenciaturas en las carreras de: Administración de Empresas, Ingeniería Agroforestal, Ingeniería en Zootecnia, Ciencias Sociales con mención en Desarrollo Local, Pedagogía, Educación Intercultural Bilingüe, Ciencias de la Educación con mención en Biología y Ciencias de la Educación con mención en Español. También se ofrecieron seis programas docentes en el nivel de Técnico Superior en Enfermería, Desarrollo Municipal, Profesor de Educación Media, Educación Intercultural Bilingüe, Pedagogía y Biología, metas de exigencia para elevar el grado académico de los docentes de cara a la acreditación y la excelencia académica.

En la extensión universitaria en Rosita se desarrolla la primera graduación de licenciados en EIB-Mayangna, en la que se graduaron 14 licenciados en EIB y 17 profesores de Educación Media en la especialidad de Español.

\section{Oferta académica 2005}

Las carreras ofertadas en esta etapa fueron en 2 modalidades de estudio, una regular donde se ofertaron Ingeniería Agroforestal, Administración de Empresas, Desarrollo Local, Ciencias de la Educación, se apertura Desarrollo Local, Pedagogía y Administración de Empresas en las extensiones de Bonanza, Rosita y Waslala. La Educación Intercultural Bilingüe en Rosita, en las modalidades: regular, sabatinos y por encuentros. En este año también se ofertaron cursos de postgrados en manejo de recursos naturales, desarrollo organizacional, planificación estratégica y técnicos superiores en desarrollo municipal.

Para la licenciatura en EIB se trasladaron las doctoras en lingüística: Elena Benedicto, María Cooks y Jane Freeland, para facilitar las clases de lengua materna en mayangna, adquisición de la segunda lengua en español y sociolingüística. El Técnico Superior en EIB estuvo conformado por estudiantes miskitus y mayangnas de las comunidades indígenas de Rosita y de Prinzapolka. 
Se obtuvo una matrícula de 887 estudiantes con una oferta de 10 carreras en pregrado: 8 en el nivel de licenciatura y 2 en Técnico Superior y se desarrollan 4 cursos de postgrado. Se facilitaron las carreras de Ciencias de la Educación, Administración de Empresas, Desarrollo Local, Educación Intercultural Bilingüe Mayangna, Ingeniería en Zootecnia e Ingeniería Agroforestal. En el nivel de Técnico Superior en Educación Media, Desarrollo Municipal y Educación Media en Educación Intercultural Bilingüe.

\section{Conclusiones}

\section{La matrícula general de URACCAN Recinto Las Minas}

Desde el 2003 se incrementó la matrícula de pre-grado de 767 estudiantes, 8o de postgrado y 736 de capacitaciones, en el caso de los postgrados y capacitaciones, estos fueron financiados por la Cooperación Austriaca.

Según las estadísticas del total de egresados de las diferentes carreras que ha ofertado URACCAN Las Minas, el 88\% se ha graduado, recibiendo el titulo correspondiente al plan de estudio culminado.

En la titulación de Técnico Superior y pre-grado, del total de graduados el 91\% tienen empleo, de los cuales el 8\% están desempeñándose en Cargos de Dirección y Políticos (Alcalde, Consejales Municipales y Regionales y Diputadas/os). Según investigación realizada por el Recinto URACCAN-Las Minas.

\section{En relación a la docencia universitaria}

El 62\% de los docentes del recinto son de tiempo horario y el 38\% de tiempo completo. El 55\% representa el sexo femenino y el $45 \%$ masculino, compromiso de género para que la mujer se desarrolle profesionalmente, y a su vez se ha avanzado en la formación especializada en postgrado y maestrías y personal es autóctono de esta región. En los cursos de postgrado, participaron 99 estudiantes de los cuales 40 (40 \%) fueron mujeres y 59 (6o \%) hombres. La realización de los cursos de postgrado, nos ha permitido establecer contactos con otros profesionales con niveles de maestría y doctorado para la realización de los diferentes encuentros programados.

\section{Incorporación del enfoque de género en la academia}

El $54 \%$ del personal administrativo del recinto son mujeres. Desde el 2001 se ha planteado un proceso inicial de sensibilización sobre el tema de Género e Interculturalidad, en las que predominaron en un $100 \%$ la participación de las mujeres en un proceso de empoderamiento. En el enfoque de género e interculturalidad, en las capitaciones y cursos de postgrado, 87 mujeres (45\%) y 105 hombres (55\%), de un total de 192. 


\section{Área experimental}

Desde la praxis del desarrollo académico, se han realizado ensayos experimentales y validaciones de variedades de granos básicos, para implementar prácticas el área de la Ingeniería Agroforestal y de apoyo a las prácticas de los cursos de postgrado. Se realizaron estudios relacionados con la planificación de una finca convencional para la transición a finca orgánica, trabajos de curso de las asignaturas: conservación de suelo y agua, diseño experimental, sociología rural, manejo integrado de plagas, pasto y forraje, topografía y edafología. Además de apoyar las prácticas de los cursos de postgrado en Recursos Naturales, monitoreo al área Biodiversidad, intercambio de experiencia con productores aledaños a la finca, intercambios de experiencia con la Universidad Nacional Agraria, con las comunidades indígenas atendidas por la ONG Pana- Pana (Waspam), producción de semilla criolla de granos básicos y de hortaliza.

\section{Investigaciones de los institutos}

El IREMADES ha realizado 16 Investigaciones en ganadería, medio ambientales; el IEPA realizó 12 Investigaciones de orden social.

En relación a la divulgación el IREMADES y el IEPA, han divulgado los estudios por medio de las radios comunitarias de URACCAN, boletín escrito EL MINERO, revista Caribe y la revista del CNU.

\section{El proceso de acompañamiento comunitario}

El IREMADES ha realizado 176 encuentros con las comunidades y estudiantes del recinto, abordando temáticas relacionadas a la agricultura orgánica, desarrollo organizacional, formulación de proyecto y manejo de ganado menor y mayor. Aproximadamente 1,889 personas fueron beneficiadas por este proyecto de extensión comunitaria. Por su parte, el IEPA, realizó 26 encuentros en las comunidades y el recinto, desarrollando la siguiente temática: Estrategia para incorporar el enfoque de género, liderazgo y trabajo en equipo, mediación comunitaria y resolución de conflictos, derechos autonómicos regionales y municipales, formulación de proyectos.

\section{Programa de becas}

La experiencia con el programa de beca del Recinto Universitario URACCAN las Minas con estudiantes de cursos regulares y por encuentro promueve las modalidades de beca monetaria y albergue, este se inicia en 1994 cuando se fundó de manera oficial la Universidad de las Regiones Autónomas de la Costa Caribe Nicaragüense.

Para la ejecución de este proyecto la Fundación para la Autonomía y Desarrollo de la Costa Atlántica de Nicaragua (FADCANIC), realizó actividades de captación y 
selección de candidatos a optar becas, entre los criterios se destacan: Presentar solicitud a FADCANIC, indicando datos básicos, los motivos por los que solicita beca y la carrera a seguir; ser originario de la Región del Atlántico Norte o cuando menos tener domicilio en la región; ser estudiantes de escasos recursos económicos con rendimiento académico mínimo de 80/100 en la Educación Secundaria, entre otros.

\section{Recomendaciones}

1. Incrementar la oferta académica de programas técnicos superiores con enfoque comunitario intercultural en alianza con las autoridades municipales, regionales, empresariales y comunitarias.

2. Incrementar las cuotas de becas para estudiantes en situación de pobreza y extrema pobreza.

3. Ampliar la incidencia de acompañamiento técnico y humanista a las comunidades por medio de los institutos adscritos a la URACCAN.

4. Elevar los indicadores de eficacia y eficiencia en el sistema universitario.

5. Mejorar el sistema de investigación-docencia y divulgar ampliamente los productos generados.

6. Potenciar los medios de comunicación en aras de la construcción de Autonomía de la Costa Caribe.

7. Consolidar el avance cuantitativo y cualitativo de conformidad al Plan Estratégico de la URACCAN.

8. Diseñar el sistema contable de los estados financieros acorde a las Normas Internacionales de Información Financiera (NIIF), para generar los estados financieros que presenten razonable y fielmente las operaciones económicas realizados por la institución en un período contable .

9. Fortalecer las capacidades de incidencia política y de alianzas en proyección a incrementar la inversión estatal en el sistema de educación en la Costa Atlántica y lograr una distribución más equitativa del presupuesto administrado por el Consejo Nacional de Universidades (CNU). 


\section{Lista de referencias}

URACCAN (2007). Sistematización del proceso de creación y desarrollo de la URACCAN en el triángulo minero 1995-2005. 1era. Edición. Siuna, Región Autónoma Atlántico Norte, Nicaragua.

Chávez Tafur, Jorge. Aprender de la experiencia. Una metodología para la sistematización. Disponible en línea: http://200. 110. 137. 61/wp/wp-content/uploads/2010/o2/ aprenderdelaexperienciajorgechavez-tafur. pdf

Estrada Ospina, Victor Mario et al. Miradas sobre la sistematización de experiencias en trabajo social. Facultad de Humanidades, Escuela de Trabajo Social y Desarrollo Humano. Disponible en línea: http: //books. google. es/books?id=nH_oH81 Cr $\mathrm{BMC} \& \mathrm{pg}=\mathrm{PA} 41 \& \mathrm{lpg}=\mathrm{PA} 41 \& \mathrm{dq}=$ metodolog\% $\mathrm{C}_{3} \% \mathrm{ADa}+\mathrm{de}+\mathrm{la}+$ sistematizaci\%C $3 \%$ B3n\&source $=$ bl\&ots=RdVo1Bgu1y\&sig $=$ LX-LqlsxQ-vGEDxnVMd8ZqgSCjo \&hl=es\&sa =X\&ei=b4D8T66cGuyC2gXLxpXxBQ\&ved=oCDoQ6AEwAQ\#v=one page $\& q=$ metodolog\% $C_{3} \% A D a \% 20$ de\%2ola\%2osistematizaci\% $C_{3} \% B 3 n \& f=f a l s e$

Hegedüs, Pedro de \& Vasallo Miguel. Sistematización de experiencias de desarrollo rural con enfoque territorial en los departamentos de Montevideo, Paysandú y Tacuarembó. Montevideo, Uruguay. Instituto Interamericano de Cooperación para la Agricultura (IICA).

IICA (2006). Red de pueblos: promoviendo el desarrollo local. Lima, Perú. Disponible en línea: http: //books. google. es/books?id=A8AFX-sojioC\&pg=PA37\&dq=metodol og\% $C_{3} \% A D a+d e+l a+$ sistematizaci\% $C_{3} \%$ B3n\&hl=es\&sa=X\&ei=hH_8T6HAHam_ 8AGK2fGVBQ\&ved=oCDoQ6AEwAQ\#v=onepage\&q=metodolog\% $C_{3} \% A D a \% 20$ de\%2ola\%2osistematizaci\% $C_{3} \%$ Bn $\& \mathrm{f}=$ false 